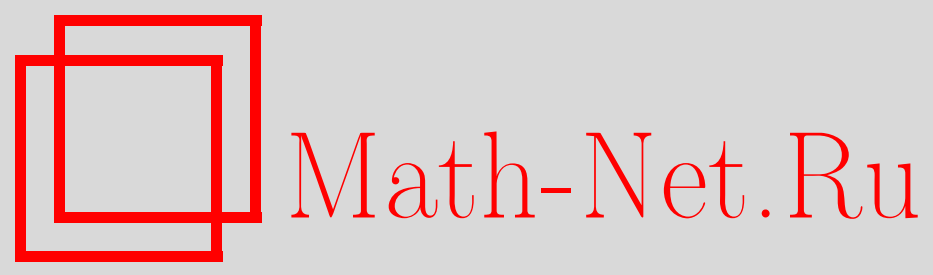

В. А. Юдин, Многомерные варианты неравенства Пэли, Матем. заметки, 2001, том 70, выпуск 6, 941-947

DOI: https://doi.org/10.4213/mzm805

Использование Общероссийского математического портала Math-Net.Ru подразумевает, что вы прочитали и согласны с пользовательским соглашением http://www . mathnet.ru/rus/agreement

Параметры загрузки:

IP: 35.173 .219 .12

26 апреля 2023 г., 16:48:28

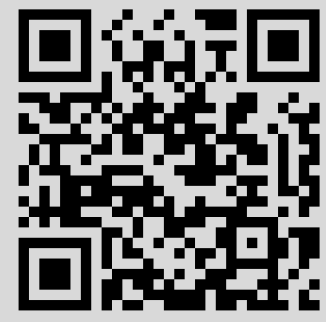




\section{МНОГОМЕРНЫЕ ВАРИАНТЫ НЕРАВЕНСТВА ПЭЛИ}

\section{В. А. Юдин}

Для тригонометрических полиномов двух переменных, имеющих спектр внутри и на границе строго выпуклой области, даются оценки снизу их норм в $L$. Они выражаются через коэффициенты Фурье, номера которых расположены на границе.

Библиограффия: 8 названий.

Пусть $T^{n}=\mathbb{R}^{n} / \mathbb{Z}^{n}-n$-мерньй тор, $x y=x_{1} y_{1}+\cdots+x_{n} y_{n}-$ скалярноепроизведение векторов $x, y$ из $\mathbb{R}^{n}$. Через

$$
\|f\|_{p}=\left\{\int_{T^{n}}|f(x)|^{p} d x\right\}^{1 / p}
$$

обозначим функции из $L_{p}\left(T^{n}\right), p \geqslant 1$, а через

$$
\tilde{f}_{\nu}=\int_{T^{n}} f(x) e^{-2 \pi i \nu x} d x
$$

- коэффициенты Фурье этих функций; sp $f=\left\{\nu \in \mathbb{Z}^{n}: \widehat{f}_{\nu} \neq 0\right\}$. Для функций из $H_{1}$ $\left(\operatorname{sp} f=\mathbb{Z}_{+}\right)$в одномерном случае известно $[1$, с. 199] неравенство Пэли

$$
\left\{\sum_{k=1}^{\infty}\left|\widehat{f}_{n_{k}}\right|^{2}\right\}^{1 / 2} \ll\|f\|_{1},
$$

где $\left\{n_{k}\right\}_{1}^{\infty}$ - лакунарная последовательность натуральных чисел. Под обозначением $A \ll B$ понимается неравенство $A \ll c B$ с константой $c$, не зависящей от $A$ и от $B$; $A \asymp B$ означает, что $A \ll B$ и $B \ll A$.

В работе [2] дано новое доказательство неравенства (1). В настоящей работе содержащийся в [2] метод получения оценок развивается на случай функций многих переменных. Через $D$ обозначим область из $\mathbb{R}^{n}$ с границей $\Gamma, \bar{D}=D \cup \Gamma$; положим

$$
f_{\Gamma}(x)=\sum_{\nu \in \Gamma} \widehat{f}_{\nu} e^{2 \pi i \nu x}, \quad f(x)=\sum_{\nu \in \bar{D}} \widehat{f}_{\nu} e^{2 \pi i \nu x}
$$

Работа выполнена при финансовой поддержке Российского фонда фундаментальных исследований, грант № 99-01-01210. 
Основной результат статьи состоит в доказательстве неравенства

$$
\left\|f_{\Gamma}\right\|_{2} \leqslant 4\|f\|_{1}
$$

для произвольной строго выпуклой области из $\mathbb{R}^{2}$. Без ограничения строгой выпуклости неравенство в общем случае неверно. Действительно, пусть на Г располагается “достаточно длинная арифметическая прогрессия"

$$
P=\{a, a \pm \mu, a \pm 2 \mu, \ldots, a \pm(q-1) \mu,\}, \quad a, \mu \in \mathbb{Z}^{n}, \quad q \in \mathbb{N} .
$$

Рассмотрим тригонометрические полиномы

$$
f^{*}(x)=f_{\Gamma}^{*}(x)=\frac{1}{q}\left|\sum_{s=0}^{q-1} e^{2 \pi i s \mu x}\right|^{2} e^{2 \pi i a x} .
$$

Нетрудно показать, что

$$
\left\|f^{*}\right\|_{1}=1, \quad\left\|f_{\Gamma}^{*}\right\|_{2} \asymp q^{1 / 2} .
$$

Следовательно, при $q \rightarrow \infty$

$$
\frac{\left\|f_{\Gamma}^{*}\right\|_{2}}{\left\|f^{*}\right\|_{1}} \rightarrow \infty
$$

Последовательности чисел $\left\{z_{k}\right\}_{1}^{\infty}, z_{k} \in \mathbb{C},\left|z_{k}\right| \leqslant 1$, поставим в соответствие последовательность комплексных чисел $\left\{w_{k}\right\}_{1}^{\infty}$ по следующему правилу:

$$
\begin{gathered}
w_{1}=z_{1}, \quad w_{2}=-\frac{\sin \alpha_{1}}{2} w_{1}^{2} \bar{z}_{2}+\cos \alpha_{1} w_{1}+\frac{\sin \alpha_{1}}{2} z_{2}, \ldots, \\
w_{k+1}=-\frac{\sin \alpha_{k}}{2} w_{k}^{2} \bar{z}_{k+1}+\cos \alpha_{k} w_{k}+\frac{\sin \alpha_{k}}{2} z_{k+1}, \ldots
\end{gathered}
$$

Для последующих оценок потребуется

УТВЕРЖДЕНИЕ 1 [3]. Для всех $k \in \mathbb{N}, \alpha_{k} \in \mathbb{R}$ для иленов последовательности (2) выполнена оценка

$$
\left|w_{k}\right| \leqslant 1 \text {. }
$$

Пусть $f(x) \in L\left(T^{n}\right), M=\left\{\nu^{(k)}\right\}_{1}^{\infty} \subset \operatorname{sp~} f \subset \mathbb{Z}^{n}$. Положим в (2)

$$
z_{1}=e^{2 \pi i \nu^{(1)} x+i \varphi_{1}}, \ldots, \quad z_{k}=e^{2 \pi i \nu^{(k)} x+i \varphi_{k}}, \ldots, \quad \widehat{f}_{\nu_{k}}=\left|\widehat{f}_{\nu_{k}}\right| e^{i \varphi_{k}}
$$

где $\varphi_{k}-$ аргумент $\widehat{f}_{\nu_{k}}$. Определим структуру спектров возникающих тригонометрических полиномов $w_{k}(x)$. Пусть $X_{k}=\mathrm{sp} w_{k}$; через

$$
A \pm B=\{c: c=a \pm b ; a \in A, b \in B\}
$$

обозначим сумму (разность) Минковского множеств $A, B$. Если $F, G$ - тригонометрические полиномы такие, что $\operatorname{sp} F=A, \operatorname{sp} G=B$, то из мультипликативных свойств тригонометрической системы функций вытекает, что

$$
\operatorname{sp} F^{2} \bar{G}=A+A-B .
$$


Следовательно, из (2), (4) находим

$$
\begin{aligned}
& X_{1}=\left\{\varnothing \cup \varnothing \cup\left\{\nu^{(1)}\right\}\right\} \text {, } \\
& X_{2}=\left\{\left[X_{1}+X_{1}-\left\{\nu^{(2)}\right\}\right] \cup X_{1} \cup\left\{\nu^{(2)}\right\}\right\} \text {, } \\
& X_{k+1}=\left\{\left[X_{k}+X_{k}-\left\{\nu^{(k+1)}\right\}\right] \cup X_{k} \cup\left\{\nu^{(k+1)}\right\}\right\},
\end{aligned}
$$

УТВЕРЖДЕНИЕ 2. Для всех $k \in \mathbb{N}, \nu \in X_{k}$ существуют иисло $s \in \mathbb{N}, 1 \leqslant s \leqslant k$, и последовательность $\left\{k_{i j}\right\} \subset \mathbb{Z}_{+}, s \leqslant i \leqslant j \leqslant k$, такие, ито

$$
\nu=\nu^{(s)}+\sum_{s \leqslant i \leqslant j \leqslant k} k_{i j}\left(\nu^{(i)}-\nu^{(j)}\right) .
$$

ДокАЗАтЕЛьСтво. При $k=1,2$ утверждение очевидно. Допустим его справедливость для $k$ и докажем его для $k+1$. Согласно $(2)$ множество $X_{k+1}$ есть объединение трех множеств $X_{k}+X_{k}-\left\{\nu^{(k+1)}\right\}, X_{k}$ и одноэлементного множества $\left\{\nu^{(k+1)}\right\}$. Если $\nu=\nu^{(k+1)}$, то утверждение справедливо. Если $\nu \in X_{k}$, то оно вытекает из предположения индукции. Пусть $\nu \in X_{k}+X_{k}-\left\{\nu^{(k+1)}\right\}$, т.е. $\nu=u+v-\nu^{(k+1)}, u, v \in X_{k}$. По предположению индукции

$$
u=\nu^{(p)}+\sum_{p \leqslant i \leqslant j \leqslant k} k_{i j}^{\prime}\left(\nu^{(i)}-\nu^{(j)}\right), \quad v=\nu^{(q)}+\sum_{q \leqslant i \leqslant j \leqslant k} k_{i j}^{\prime \prime}\left(\nu^{(i)}-\nu^{(j)}\right) .
$$

Складьвая, найдем

$$
\begin{aligned}
\nu & =\nu^{(p)}+\nu^{(q)}-\nu^{(k+1)}+\sum_{p \leqslant i \leqslant j \leqslant k} k_{i j}^{\prime}\left(\nu^{(i)}-\nu^{(j)}\right)+\sum_{q \leqslant i \leqslant j \leqslant k} k_{i j}^{\prime \prime}\left(\nu^{(i)}-\nu^{(j)}\right) \\
& =\nu^{(r)}+\sum_{r \leqslant i \leqslant j \leqslant k+1} k_{i j}\left(\nu^{(i)}-\nu^{(j)}\right), \quad k_{i j} \in \mathbb{Z}_{+}, \quad r=\min \{p, q\} .
\end{aligned}
$$

Имеет место

ТЕОРемА. Пусть для $f(x) \in L\left(T^{n}\right)$ выполняются условия

1) $M \subset \operatorname{sp} f$

2) для всех $k \in \mathbb{N} \quad X_{k}+X_{k}-\left\{\nu^{(k+1)}\right\} \cap \operatorname{sp} f=\varnothing$.

Тогда

$$
\left\{\sum_{\nu \in M}\left|\widehat{f}_{\nu}\right|^{2}\right\}^{1 / 2} \leqslant 2\|f\|_{1}
$$


ДокаЗАТЕльСтво. Выберем произвольное $k \in \mathbb{N}$ и проинтегрируем $\bar{f}(x)$ с весом $w_{k+1}(x)$ на $T^{n}$. Имеем

$$
I_{k+1}=\int_{T^{n}} \bar{f}(x) w_{k+1}(x) d x .
$$

Из (3) найдем

$$
\left|I_{k+1}\right|=\left|\int_{T^{n}} \bar{f}(x) w_{k+1}(x) d x\right| \leqslant\|f\|_{1}\left\|w_{k+1}\right\|_{\infty} \leqslant\|f\|_{1} .
$$

Из (2) получим

$$
\begin{aligned}
I_{k+1}= & -\frac{\sin \alpha_{k}}{2} \int_{T^{n}} \bar{f}(x) w_{k}^{2}(x) \bar{z}_{k+1}(x) d x \\
& +\cos \alpha_{k} \int_{T^{n}} \bar{f}(x) w_{k}(x) d x+\frac{\sin \alpha_{k}}{2} \int_{T^{n}} \bar{f}(x) z_{k+1}(x) d x .
\end{aligned}
$$

По условию 2) теоремы

$$
\operatorname{sp} w_{k}^{2} \bar{z}_{k+1} \cap \operatorname{sp} f=\varnothing,
$$

следовательно, по равенству Парсеваля первое слагаемое обращается в 0 , а

$$
\int_{T^{n}} \bar{f}(x) z_{k+1}(x) d x=\left|\widehat{f}_{\nu_{k+1}}\right|
$$

В итоге приходим к рекуррентной формуле

$$
I_{k+1}=\frac{\sin \alpha_{k}}{2}\left|\widehat{f}_{\nu_{k+1}}\right|+\cos \alpha_{k} I_{k} .
$$

Выберем угол $\alpha_{k}$ так, чтобы правая часть (8) стала максимальной:

$$
\sin \alpha_{k}=\frac{\left|\widehat{f}_{\nu_{k+1}}\right| / 2}{\sqrt{\left|\widehat{f}_{\nu_{k+1}}\right|^{2} / 4+I_{k}^{2}}}, \quad \cos \alpha_{k}=\frac{I_{k}}{\sqrt{\left|\widehat{f}_{\nu_{k+1}}\right|^{2} / 4+I_{k}^{2}}} .
$$

Из (8) получаем рекуррентное соотношение

$$
I_{k+1}^{2}=\frac{1}{4}\left|\widehat{f}_{\nu_{k+1}}\right|^{2}+I_{k}^{2}
$$

откуда

$$
I_{k+1}^{2}=\frac{1}{4}\left(\left|\widehat{f}_{\nu_{k+1}}\right|^{2}+\cdots+\left|\widehat{f}_{\nu_{2}}\right|^{2}\right)+\left|\widehat{f}_{\nu_{1}}\right|^{2} \geqslant \frac{1}{4} \sum_{s=1}^{k+1}\left|\widehat{f}_{\nu_{s}}\right|^{2} .
$$

Используя неравенство (7), находим

$$
\frac{1}{2}\left\{\sum_{s=1}^{k+1}\left|\widehat{f}_{\nu_{s}}\right|^{2}\right\}^{1 / 2} \leqslant\|f\|_{1}
$$

устремляя $k \rightarrow \infty$, имеем (6). Теорема доказана. 
СлеДСтвИЕ 1. Пусть $n=2 u$ Г - гранича строго выпуклой области D. Тогда

$$
\left\|f_{\Gamma}\right\|_{2} \leqslant 4\|f\|_{1}
$$

ДокаЗАТЕЛьСтво. Проведем к $D$ четыре опорные прямые: две вертикальные и две горизонтальные. Ввиду строгой выпуклости каждая прямая имеет с Г лишь одну общую точку. Полученные четыре точки разобьют кривую $\Gamma$ на четыре части $\Gamma_{1}, \Gamma_{2}, \Gamma_{3}$, $\Gamma_{4}$. Будем считать, что $\Gamma_{1}$ ограничена точками $A$ и $B$. Точка $A$ лежит на нижней опорной прямой, а $B$ лежит на правой вертикальной прямой. Занумеруем целые точки из $\Gamma_{1}$, двигаясь по $\Gamma_{1}$ в направлении от $A$ к $B$. Их совокупность обозначим через $M$. Так как $\Gamma_{1}$ - график строго выпуклой вниз функции, то отношения разностей возрастают. Из представления (5) вытекает, что если хотя бы один коэффициент $k_{i j} \neq 0$, то $\nu \notin \bar{D}$. Таким образом,

$$
\forall k \in \mathbb{N} \quad X_{k}+X_{k}-\nu^{(k+1)} \cap \bar{D}=\varnothing .
$$

Из (6) получим

$$
\left\|f_{\Gamma_{1}}\right\|_{2}^{2} \leqslant 4\|f\|_{1}^{2}
$$

аналогично устанавливаем

$$
\left\|f_{\Gamma_{i}}\right\|_{2}^{2} \leqslant 4\|f\|_{1}^{2}, \quad i=2,3,4
$$

Складывая, найдем

$$
\left\|f_{\Gamma}\right\|_{2}^{2}=\left\|f_{\Gamma_{1}}\right\|_{2}^{2}+\cdots+\left\|f_{\Gamma_{4}}\right\|_{2}^{2} \leqslant 16\|f\|_{1}^{2}
$$

откуда следует (9).

Отметим, что в частном случае, когда весь спектр $f$ содержится на строго вьпуклой кривой $f=f_{\Gamma}$, неравенство Кука [4], [5] $\|f\|_{4} \ll\|f\|_{2}$ приводит к более сильной оценке $\|f\|_{4} \ll\|f\|_{1}$.

Оценки, подобные (9), иногда справедливы и в ситуациях, когда лишь часть границы $D$ является строго выпуклой кривой. Рассмотрим один пример. Пусть функции $\varphi(t), \psi(t)$ определены на отрезке $[a, b], a, b \in \mathbb{Z}$, и $1+\varphi(t) \leqslant \psi(t), t \in[a, b]$. Положим

$$
\begin{aligned}
\bar{D} & =\left\{\nu \in \mathbb{Z}^{2}: a \leqslant \nu_{1} \leqslant b, \varphi\left(\nu_{1}\right) \leqslant \nu_{2} \leqslant \psi\left(\nu_{1}\right)\right\}, \\
\Gamma & =\left\{\nu \in \mathbb{Z}^{2}: a \leqslant \nu_{1} \leqslant b, \nu_{2}=\left[\varphi\left(\nu_{1}\right)\right]+1\right\},
\end{aligned}
$$

где $[x]$ - целая часть $x$.

СледСтвиЕ 2. Пусть функиия $\varphi(t)$ дважды дифференцируема на $(a, b)$, причем $\varphi^{\prime \prime}(t) \geqslant \lambda>0, t \in(a, b)$. Тогдa

$$
\frac{1}{2}\left(\frac{\sqrt{\lambda}}{\sqrt{\lambda}+2}\right)^{1 / 2}\left\|f_{\Gamma}\right\|_{2} \leqslant\|f\|_{1}
$$


ДокАЗАТЕльСтво. Все множество $Г$ разобьем на $q=1+[\sqrt{2 / \lambda}]$ частей:

$$
\Gamma_{j}=\{a+j+k q,[\psi(a+j+k q)]+1\}, \quad j=0,1, \ldots, q-1, \quad k=0,1, \ldots,\left[\frac{b-a}{q}\right] .
$$

При каждом $j$ оценим снизу вторые разности:

$$
\begin{aligned}
I_{j} & =[\varphi(a+j+(k-1) q)]-2[\varphi(a+j+k q)]+[\varphi(a+j+(k+1) q)] \\
& \geqslant \varphi(a+j+(k-1) q)-2 \varphi(a+j+k q)+\varphi(a+j+(k+1) q)-2 .
\end{aligned}
$$

Так как

$$
\varphi(t-h)-2 \varphi(t)+\varphi(t+h)=h^{2} \varphi^{\prime \prime}(\bar{t}), \quad \bar{t} \in(-h, h),
$$

то

$$
I_{j} \geqslant q^{2} \lambda-2=\left(1+\left[\sqrt{\frac{2}{\lambda}}\right]\right)^{2} \lambda-2>0 .
$$

Таким образом, все целые точки $\Gamma_{j}$ располагаются на строго вьпуклой кривой. Следовательно, вся последовательность множеств

$$
X_{k}+X_{k}-\{a+j+(k+1) q,[\varphi(a+j+(k+1) q)]+1\}, \quad k=1,2, \ldots,
$$

соответствуюшая $\Gamma_{j}$, не пересекается с $D$. Согласно $(6)$ имеем

$$
\|f\|_{1}^{2} \geqslant \frac{1}{4}\left\|f_{\Gamma_{j}}\right\|_{2}^{2}, \quad j=0,1, \ldots, q-1 .
$$

Складывая, найдем

$$
\|f\|_{1}^{2} \geqslant \frac{1}{4 q} \sum_{j=0}^{q-1}\left\|f_{\Gamma_{j}}\right\|_{2}^{2}=\frac{1}{4 q}\left\|f_{\Gamma}\right\|_{2}^{2}=\frac{1}{4} \frac{\sqrt{\lambda}}{\sqrt{\lambda}+\sqrt{2}}\left\|f_{\Gamma}\right\|_{2}^{2},
$$

откуда следует (10).

Оценка (10) не зависит от вида функции $\psi(t)$, т.е. от формы верхней границы $D$. В частности, пусть

$$
\bar{D}_{N}=\left\{\nu \in \mathbb{Z}^{2}: 0 \leqslant \nu_{1} \leqslant N, \nu_{1}^{2} \leqslant \nu_{2} \leqslant N^{2}\right\}, \quad \Gamma=\left\{\nu \in \mathbb{Z}^{2}: 0 \leqslant \nu_{1} \leqslant N, \nu_{2}=\nu_{1}^{2}\right\} .
$$

Здесь $\varphi(t)=t^{2}, \varphi^{\prime \prime}(t)=2=\lambda, \psi(t)=N^{2}$. Все коэффициенты Фурье тригонометрического полинома $f(x)$ положим равными 1. Из (10) найдем

$$
\|f\|_{1}=\int_{T^{n}}\left|\sum_{\nu \in \bar{D}_{n}} e^{2 \pi i \nu x}\right| d x \gg\left\|f_{\Gamma}\right\|_{2}=\left\{\sum_{\nu \in \Gamma} 1\right\}^{1 / 2} \gg N^{1 / 2} .
$$


Тем самым, получена оценка снизу $N^{1 / 2}$ для нормы в $L_{1}$ ядра Дирихле, соответствующего $\bar{D}_{N}$. Из анизотропной оценки [6], [7]

$$
\|f\|_{1} \ll L_{1}^{1 / 2} \ln \left(\frac{L_{2}}{L_{1}}+1\right)
$$

где $L_{1}$ - толщина $\bar{D}_{N}$ в направлении оси $O X_{2}\left(L_{1} \asymp N\right)$, a $L_{2}-$ толщина $\bar{D}_{N}$ в направлении оси $O X_{1}\left(L_{2} \asymp N^{2}\right)$, находим

$$
\|f\|_{1} \ll N^{1 / 2} \ln N
$$

Верхняя и нижняя оценка различаются лишь на логарифмический множитель. Однако использование неравенств (9), (10) для оценки константы Лебега, порожденной кругом, приводит к плохим оценкам снизу. Неравенства (9), (10) "хороши", когда "достаточно большая" часть спектра $f$ сосредоточена на границе.

В заключение отметим, что в статье использовались соображения из [2], однако возможно применение более ранних результатов [8]. Благодарю С. В. Конягина за полезные рассуждения.

\section{СПИСОК ЦИТИРОВАННОЙ ЛИТЕРАТУРЫ}

[1] Зигмунд А. Тригонометрические ряды. Т. II. М.: Мир, 1965.

[2] Smith B. Two trigonometric designs: one-sided Riesz products and Littlewood products. General inequalities 3. // Intern. Ser. of Numer. Math. 1983. V. 64. P. 141-148.

[3] Юдин В. А. Интегральные нормы тригонометрических полиномов // Матем. заметки. 2001. T. 70. № 2. C. $308-315$.

[4] Cooke R. A Cantor-Lebesgue theorem in two dimensions // Proc. Amer. Math. Soc. 1971. V. 30. P. 547-550.

[5] Алимов Ш. А., Ильин В. А., Никишин Е. М. Вопросы сходимости кратных тригонометрических рядов и спектральных разложений. I // УМН. 1976. V. 31. №6. P. 28-83; II // УМН. 1977. V. 32. №1. Р. 107-130.

[6] Подкорытов А. Н. О константах Лебега двойных рядов Фурье // Вест. ЛГУ. 1977. № 7. C. $79-84$.

[7] Юдин А. А., Юдин В. А. Дискретные теоремы вложения и константы Лебега // Матем. заметки. 1977. Т. 22. №3. С. 381-394.

[8] Fournier J. F. On a theorem of Paley and the Littlewood conjecture // Archiv für Math. 1979. V. 17. P. $199-216$. 